\title{
Can 5-mm Axial CT Images Sufficiently Depict the Hyperdense Middle Cerebral Artery Sign in Patients with Acute Brain Ischemia?*
}

\author{
Tomoya Nakatsuka\#, Mamoru Kurotsuchi" ${ }^{\#}$ Hideo Morita, Tsutomu Inaoka, Hitoshi Terada ${ }^{\dagger}$ \\ Department of Radiology, Toho University Sakura Medical Center, Sakura, Japan \\ Email: †terada@sakura.med.toho-u.ac.jp
}

Received October 27, 2013; revised November 27, 2013; accepted December 4, 2013

Copyright (C) 2013 Tomoya Nakatsuka et al. This is an open access article distributed under the Creative Commons Attribution License, which permits unrestricted use, distribution, and reproduction in any medium, provided the original work is properly cited. In accordance of the Creative Commons Attribution License all Copyrights $\mathbb{C} 2013$ are reserved for SCIRP and the owner of the intellectual property Tomoya Nakatsuka et al. All Copyright (C) 2013 are guarded by law and by SCIRP as a guardian.

\begin{abstract}
The purpose of this study was to determine whether 5-mm axial CT images can sufficiently depict the hyperdense middle cerebral artery (MCA) sign in patients with acute brain ischemia. Materials and Methods: A retrospective review of 92 cases of ischemic brain infarction confirmed nine patients showing hyperdense MCA signs. CT images were acquired on a 64-slice helical scanner. Images were reconstructed into contiguous 5-mm axial, coronal and sagittal datasets. The first CT scan images of the patients with hyperdense MCA signs and an equal number of CT scans without hyperdense MCA signs inserted at random were analyzed in a blinded review. The presence of the hyperdense MCA sign in M1 segments was recorded. Round regions of interest (ROI) were placed over the M1 segments of the MCAs and the attenuation values in Hounsfield units (HU) were measured on the sagittal and axial images. Results: Nine patients showed a hyperdense MCA sign. They consisted of 4 male and 5 female (mean age, 74.3 years; age range, $45-88$ years). On the blinded review, hyperdense MCA signs were detected on axial images in 7 patients. In four of the 7 patients, the hyperdense MCA sign was more conspicuous on sagittal images than that on axial images. Hyperdense MCA sign was detected on sagittal images of all 9 patients and exclusive to the sagittal images in two patients. The ROI study showed higher attenuation for the affected MCA on sagittal images (46 - $65 \mathrm{HU})$ than that on axial images (36 - $54 \mathrm{HU})$. Conclusion: In patients with acute brain ischemia, $5-\mathrm{mm}$ axial CT images cannot sufficiently depict the hyperdense MCA sign. Sagittal images may be superior to axial images for identifying the sign.
\end{abstract}

Keywords: Multidetecter CT; Hyperdense MCA Sign; Sagittal Reformation

\section{Introduction}

Over the last three decades, the hyperdense middle cerebral artery (MCA) sign has been confirmed as an indicator of acute ischemic change [1-4]. This sign is not only useful in the diagnosis of MCA occlusion, but also as a predictor of prognosis and treatment [2-6].

Since the development of multirow-detector CT (MDCT), the advantages of helical acquisition modality and multirow-detector technology have increased, for example, higher image quality, reduced irradiation and shortened acquisition time $[7,8]$. There is also literature that indi-

\footnotetext{
"The authors declare that they have no conflict of interest.

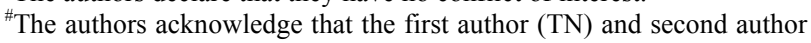
(MK) contributed equally to this paper.

${ }^{\dagger}$ Corresponding author.
}

cates further improvement in image quality and reduction of image artifacts if the number of detectors in the CT system is increased [9]. Furthermore, the MDCT allows some advantages in multiplaner reformation (MPR), maximum intensity projection (MIP), volume-rendered reconstruction, and three-dimensional reconstruction (3D) [10]. In our hospital, we usually use these new techniques for all head CT examinations as standard, especially when making MPR views for evaluations, even for patients coming to the emergency room. Sometimes we notice that we can find the hyperdense MCA sign more easily in sagittal views than axial views.

To date, there are no systematic studies investigating the usefulness of sagittal CT images for identifying the hyperdense MCA sign in stroke patients. So we investi- 
gated whether sagittal CT images are more useful for identifying the hyperdense MCA sign in acute ischemic patients than axial $\mathrm{CT}$ images.

\section{Materials and Methods}

\subsection{Patient Selection}

We retrospectively reviewed the records of 266 consecutive patients who were admitted to our institute because of clinical symptoms of ischemic brain infarction from June 1, 2007, to August 31, 2009. Records were compiled from our hospital's database. Next, we obtained the histories of these 266 patients from the medical records. Nonenhanced CT images were available for 251 of the 266 patients. The histories of these 251 patients were reviewed for compliance with both of the following inclusion criteria: (a) the first CT scan was obtained within 24 hours after onset of symptoms, (b) the final clinical diagnosis of acute cerebral infarction was confirmed by follow-up CT scan or MRI. The histories of 92 of the 251 patients satisfied these criteria. These 92 patients include 60 male (65.2\%) and 32 female (34.8\%); aged 29 - 93 years, mean age 70.5 years.

The first CT scans of the 92 selected patients were reevaluated for the presence of a hyperdense MCA sign by two board certified radiologists in consensus. The presence of a hyperdense MCA sign was defined using the following criteria: (a) appearance of partial or diffuse higher density in M1 segment of the MCA than other vascular structures on the first nonenhanced $\mathrm{CT}$ scan, (b) clinical symptoms of infarction in the corresponding MCA territory, (c) disappearance of a unilateral hyperdense MCA on the follow-up nonenhanced CT scan. All three orthogonal datasets were evaluated in this evaluation. The hyperdense MCA sign was confirmed in nine of the 92 patients $(9.8 \%)$.

Nine patients with hyperdense MCA sign consisted of 4 men and 5 females (aged $45-88$ years, mean age 74.3 years). The remaining 83 patients did not show the hyperdense MCA sign despite including clear cases of atherothrombotic infarction, lacunar infarction, branch atheromatous disease, infarction in the posterior circulation territory, and cardioembolic ICA occlusion or cardioembolic MCA occlusion. The histories of the nine patients revealed that; 7 patients had atrial fibrillation (77.7\%), three cerebral infarction (33.3\%), three diabetes (33.3\%), one hypertension (11.1\%), one atrioventricular block post pacemaker operation (11.1\%), and one sick sinus syndrome post pacemaker operation (11.1\%). Seven of the 9 patients were hemiplegic at onset, and the remaining two patients were unconscious.

Six of the 9 patients had MRA examinations within 24 hours following the first CT scan. MRA confirmed MCA occlusion in 3 cases and internal carotid artery (ICA) occlusion in 3 cases corresponding to the hyperdense MCA signs. Two of the 9 patients had a conventional angiography within 3 hours following the first CT scan. Conventional angiography confirmed MCA occlusion in one case and ICA occlusion in the other case corresponding to hyperdense MCA sign. One of the nine patients did not undergo MRA or conventional angiography.

\subsection{Scanning Procedure}

CT images were acquired on a 64-slice helical scanner (Aquilion, Toshiba medical systems, Otawara, Japan) using our standard protocol for the brain in the adults: helical scanning, $120-\mathrm{kV}, 300-\mathrm{mAs}$, 1s rotation time, 64 $\times 0.5 \mathrm{~mm}$ collimation, 0.641 pitch factor, field of view $22.03 \mathrm{~cm}$, matrix $512 \times 512$. Images were reconstructed into contiguous 5-mm axial, coronal and sagittal datasets as part of routine clinical care. The window width and window level were fixed as $90 \mathrm{HU}$ and $40 \mathrm{HU}$ respecttively in all image evaluation. All images were reviewed using a Rapid-eye viewer system (Toshiba medical systems, Otawara, Japan).

\subsection{Blind Review}

The first CT scans of the nine patients with confirmed hyperdense MCA signs were added to an equivalent number of CT scans without hyperdense MCA signs, inserted in random order. This enriched study set was divided into axial, coronal and sagittal images and analyzed separately by two radiologists in consensus. This review was performed by two different radiologists who had no access to the results of patient selection. Axial images were reviewed first, followed by separate reviews of the coronal and sagittal images. The two readers were blinded to the results of each other image series. So when reading the sagittal images the readers were blinded to the results of the axial and coronal series. Consensus was always achieved. The presence of any hyperdense MCA sign was recorded. The hyperdense MCA sign was evaluated according to three categories, No hyperdense MCA sign (-); a probable MCA sign (+); and a definite MCA sign (++).

\subsection{Region of Interest Analysis}

Measurements were taken on a Rapid-eye viewer system. Round regions of interest (ROI) of $2 \mathrm{~mm}^{2}$ were placed over the M1 segments of MCAs and the attenuation values in Hounsfield units (HU) were measured. The ROIs were taken on the sagittal and axial images. Care was taken in particular to ensure that the boundaries of ROI did not extend beyond the merging of the arteries. The densest segments of the visualized arteries were measured to minimize the partial volume effect. All measure- 
ments were made by two radiologists in consensus. Statistical analyses were conducted using paired t test.

The Institutional Review Board approved the study (IRB \#: 2010-017).

\section{Results}

On the blind review (see Table 1), we confirmed the presence of hyperdense MCA sign on axial images in 7
(77.8\%) patients, on coronal images in $6(66.7 \%)$ patients, and on sagittal images in all $9(100 \%)$ patients. In four of the 7 patients $(71.4 \%)$, the hyperdense MCA sign was more conspicuous on sagittal images than on axial images. Hyperdense MCA signs were detected exclusively on sagittal images in two of the 9 patients (22.2\%) (Figure 1). There were no false-positive cases. (No hyperdense MCA sign was detected on any of the CT images
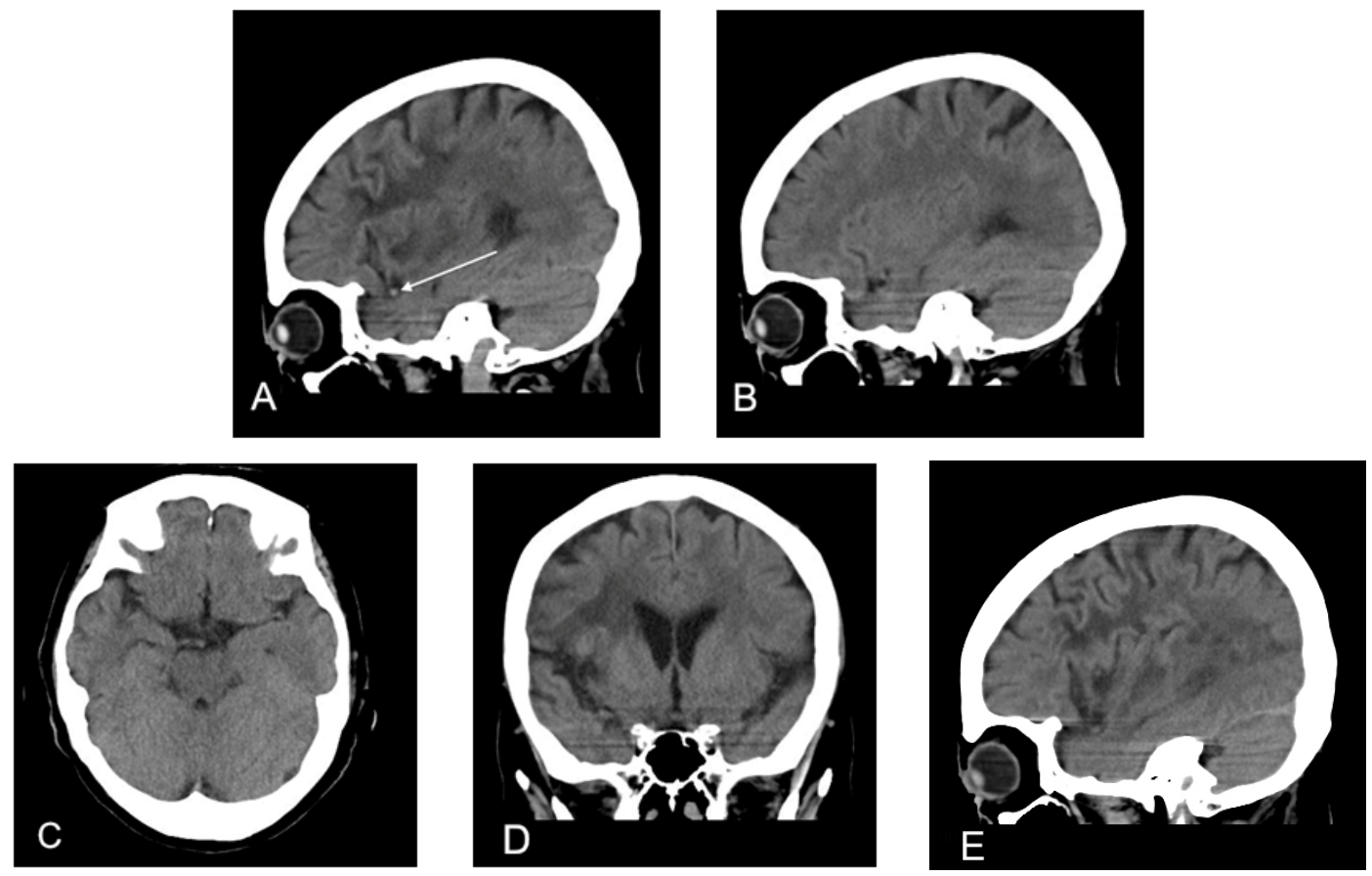

Figure 1. MRA proven right MCA occlusion. The patient has a past history of right MCA territory infarction. The right parasagittal image 14 hours after the onset (A) shows hyperdense MCA sign (MCA sign +); Left parasagittal image (B); axial image (C); and coronal image (D) show no hyperdense MCA sign (MCA sign -); The right parasagittal image 11 hours later (E) shows the disappearance of the hyperdense MCA sign.

Table 1. Summary of cases with hyperdense MCA sign.

\begin{tabular}{|c|c|c|c|c|c|c|c|}
\hline \multirow[b]{2}{*}{ Case $^{\#}$} & \multirow[b]{2}{*}{ Coronal } & \multirow[b]{2}{*}{ Axial } & \multirow[b]{2}{*}{ Sagittal } & \multicolumn{2}{|c|}{ Vascular image \& Occluded artery } & \multirow{2}{*}{$\begin{array}{c}\text { Mean HU of ROI } \\
\text { Axial Images }\end{array}$} & \multirow{2}{*}{$\begin{array}{c}\text { Mean HU of ROI } \\
\text { Sagittal Images }\end{array}$} \\
\hline & & & & $M R A$ & DSA & & \\
\hline 1 & - & - & + & Lt. ICA & ND & $40 / 36$ & $53 / 41$ \\
\hline 2 & - & - & + & Rt. MCA & ND & $36 / 37$ & $46 / 38$ \\
\hline 3 & - & + & + & Rt. MCA & ND & $42 / 33$ & $51 / 38$ \\
\hline 4 & + & + & + & Rt. ICA & ND & $54 / 43$ & $65 / 31$ \\
\hline 5 & + & + & + & Rt. MCA & ND & $52 / 42$ & $49 / 45$ \\
\hline 6 & + & + & ++ & Rt. ICA & ND & $49 / 34$ & $51 / 39$ \\
\hline 7 & + & + & ++ & ND & Lt. ICA & $45 / 30$ & $54 / 36$ \\
\hline 8 & + & + & ++ & ND & Lt. MCA & $42 / 34$ & $64 / 36$ \\
\hline 9 & + & + & ++ & ND & ND & $45 / 40$ & $52 / 41$ \\
\hline
\end{tabular}

-: no HDMCA sign, +: probable HDMCA sign, ++: definite HDMCA sign, ND: not done, MRA: magnetic resonance angiography, DSA: digital subtraction angiography, Lt.: left, Rt.: right, ICA: internal carotid artery, MCA: middle cerebral artery, HU: Hounsfield unit, ROI: region of interest. 
without hyperdense MCA signs that were inserted into the blind studies).

Affected MCAs on axial images had a mean attenuation ranging 36 - $54 \mathrm{HU}$ versus 30 - $43 \mathrm{HU}$ for unaffected vessels. Consequently attenuation values for the occluded vessels and those not occluded overlapped significantly (between 36 - $43 \mathrm{HU}$ ). While on sagittal images affected MCAs had a mean attenuation ranging 46 - 65 HU versus 31 - 45 HU for unaffected vessels. There was no overlap in attenuation values for occluded vessels with those not occluded (Figure 2). The mean attenuation of affected MCAs was significantly higher on sagittal images than on axial images $(p<0.005)$. There was no significant difference between the mean attenuation of unaffected MCAs on sagittal images and axial images $(p=0.36)$.

\section{Discussion}

Some papers describe a "MCA dot sign" as a hyperdensity in the M2 and M3 segments of the MCA seen in the sylvian fissure on axial images [11,12]. Another report verified the validation of this sign with an angiographic correlation study [13]. The dot sign is a highly specific indicator of acute thrombus, and a useful additional acute stroke CT marker. The occluded M2 and M3 segments lie perpendicular to the axial plane. We think it important that the occluded vessel is viewed in cross section to reduce the partial volume effect. All of our cases had the hyperdense MCA sign in the M1 segment of the middle cerebral artery which runs horizontally, perpendicular to the sagittal view. So we see the hyperdense MCA sign as a "dot sign" on sagittal views. We think this explains the difference in the incidence of the hyperdense MCA sign on sagittal and axial slices.

There is a problem of false positive results [14]. An increased hematocrit, residual contrast medium from previous examinations and partial volume averaging ar-

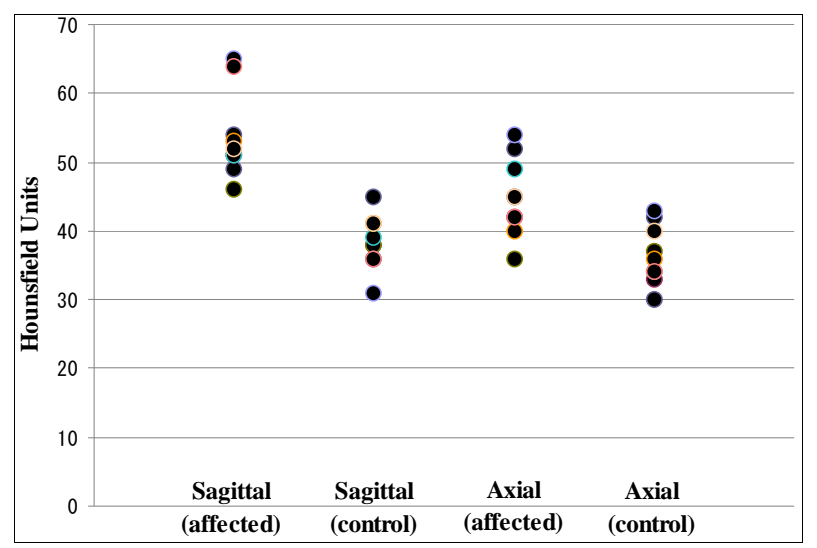

Figure 2. The distribution of mean HU values of ROIs on MCA. (affected): affected MCA, (control): non-affected MCA. tifacts from calcification in arterial walls are well recognized causes of false positive results. One study reported that they could distinguish a true hyperdense middle cerebral artery sign from the pseudohyperdense MCA sign by measuring the absolute attenuation value of abnormal MCA [15]. The study confirmed that if the absolute attenuation of the affected vessel is higher than 43 Hounsfield units (HU), the MCA sign is true for occlusion. In our cases, there was no overlap of attenuation value between affected and unaffected MCA on sagittal images, while there was overlap of attenuation value between affected and unaffected MCA on axial images. We think this overlap on axial images is due to partial volume averaging artifact from the surrounding cerebrospinal fluid. Sagittal reconstruction allows us to assess the M1 segment in cross section reducing partial volume averaging artifacts from surrounding cerebrospinal fluid and calcification in the arterial wall.

One paper described the usefulness of varying window parameters and a maximum-intensity projection (MIP) to detect dense intracranial arteries [16]. They found dense cerebral arteries in $21 \%$ of standard images, $42 \%$ with a narrow window and $78 \%$ on the thin slices in patients with a worse outcome. However, as they describe, this result was obtained from reviewing images made on a workstation. We used thick, routine CT images with an ordinary viewer and fixed window width/center, not on a workstation.

The main limitation of our study is the small number of cases. Moreover our study is retrospective in design. Further rigorous prospective assessment of the effectiveness of sagittal reconstruction to detect the hyperdense MCA sign in a larger case series is mandatory. In our study, however, axial images showed no hyperdense MCA sign in two of the nine cases (Figure 2). This result suggests there is a risk of overlooking the hyperdense MCA sign in many clinical situations if only axial images are viewed. We believe routinely reconstructed sagittal views are important to help us find the hyperdense MCA sign in daily clinical work especially in the emergency room.

In conclusion, we state that sagittal reformation using multislice helical CT helps to detect the hyperdense MCA sign in acute stroke.

\section{REFERENCES}

[1] G. Gacs, A. J. Fox, H. J. M. Barnett and F. Vinuela, "CT Visualization of Intracranial Arterial Thromboembolism," Stroke, Vol. 14, No. 5, 1983, pp. 756-762. http://dx.doi.org/10.1161/01.STR.14.5.756

[2] T. A. Tomsick, T. G. Brott, C. P. Olinger, W. Barsan, J. Spilker, R. Eberle, et al., "Hyper Dense Middle Cerebral Artery: Incidence and Quantitative Significance," Neuroradiology, Vol. 31, No. 4, 1989, pp. 312-315. 
http://dx.doi.org/10.1007/BF00344173

[3] S. Bastianello, A. Pierallini, C. Colonnese, G. Brughitta, U. Angeloni, M. Antonelli, et al., "Hyperdense Middle Cerebral Artery CT Sign: Comparison with Angiography in the Acute Phase of Ischemic Supratentorial Infarction," Neuroradiology, Vol. 33, No. 3, 1991, pp. 207-211. http://dx.doi.org/10.1007/BF00588219

[4] D. Leys, J. P. Pruvo, O. Godefroy, P. Randepierre and X. Leclerc, "Prevalence and Significance of Hyperdense Middle Cerebral Artery in Acute Stroke," Stroke, Vol. 23, No. 3, 1992, pp. 317-324. http://dx.doi.org/10.1161/01.STR.23.3.317

[5] C. Manelfe, V. Larrue, R. von Kummer, L. Bozzao, P. Ringleb, S. Bastianello, et al., "Association of Hyperdense Middle Cerebral Artery Sign with Clinical Outcome in Patients Treated with Tissue Plasminogen Activator," Stroke, Vol. 30, No. 4, 1999, pp. 769-772. http://dx.doi.org/10.1161/01.STR.30.4.769

[6] D. M. Somford, P. J. Nederkoorn, D. R. Rutgers, L. J. Kappelle, W. P. T. M. Mali and J. van der Grond, "Proximal and Distal Hyperattenuating Middle Cerebral Artery Signs at CT: Different Prognostic Implications," Radiology, Vol. 223, No3, pp. 667-671. http://dx.doi.org/10.1148/radiol.2233011017

[7] D. Hernalsteen, G. Cosnard, A. Robert, C. Grandin, A. Vlassenbroek and T. Duprez, "Suitability of Helical Multislice Acquisition Technique for Routine Unenhanced Brain CT: An Image Quality Study Using a 16-Row Detector Configuration," European Radiology, Vol. 17, No. 4, 2007, pp. 975-982. http://dx.doi.org/10.1007/s00330-006-0360-9

[8] T. R. Jones, R. T. Kaplan, B. Lane, S. W. Atlas and G. D. Rubin, "Single- versus Multi-Detector Row CT of the Brain: Quality Assessment," Radiology, Vol. 219, No. 3, 2001, pp. 750-755. http://dx.doi.org/10.1148/radiology.219.3.r01jn47750

[9] B. Ertl-Wagner, L. Eftimov, J. Blume, R. Bruening, C. Becker, J. Cormack, et al., "Cranial CT with 64-, 16-, 4-, and Single-Slice CT Systems-Comparison of Image Qua- lity and Posterior Fossa Artifacts in Routine Brain Imaging with Standard pRotocols," European Radiology, Vol. 18, No. 8, 2008, pp. 1720-1726. http://dx.doi.org/10.1007/s00330-008-0937-6

[10] G. T. Flohr, S. Schaller, K. Stierstorfer, H. Bruder, B. M. Ohnesorge and U. J. Schoepf, "Multi-Detector Row CT Systems and Image-Reconstruction Techniques," Radiology, Vol. 235, No. 3, 2005, pp. 756-773. http://dx.doi.org/10.1148/radiol.2353040037

[11] P. A. Barber, A. M. Demchuk, M. E. Hudon, J. H. W. Pexman, M. D. Hill and A. M. Buchan, "Hyperdense Sylvian Fissure MCA "Dot" Sign; A CT Maker of Acute Ischemia," Stroke, Vol. 32, No. 1, 2001, pp. 84-88. http://dx.doi.org/10.1161/01.STR.32.1.84

[12] S. K. Shelty, "The MCA Dot Sign," Radiology, Vol. 241, No. 1, 2006, pp. 315-318. http://dx.doi.org/10.1148/radiol.2411040573

[13] M. C. Leary, C. S. Kidwell, J. P. Villablanca, S. Starkman, R. Jahan, G. R. Duckwiler, et al., "Validation of Computed Tomographic Middle Cerebral Artery 'Dot' Sign: An Angiographic Correlation Study," Stroke, Vol. 34, No. 11, 2003, pp. 2636-2640. http://dx.doi.org/10.1161/01.STR.0000092123.00938.83

[14] R. A. Rauch, C. Bazan 3rd, E. M. Larsson and J. R. Jinkins, "Hyperdense Middle Cerebral Arteries Identified on CT as a False Sign of Vascular Occlusion," American Journal of Neuroradiology, Vol. 14, No. 3, 1993, pp. 669-673.

[15] C. K. Koo, E. Teasdale and K. W. Muir, "What Constitutes a True Hyperdense Middle Cerebral Artery Sign?" Cerebrovascular Diseases, Vol. 10, No. 6, 2000, pp. 419423. http://dx.doi.org/10.1159/000016101

[16] D. Gadda, L. Vannucchi, F. Niccolai, A. T. Neri, L. Carmignani and P. Pacini, "CT in Acute Stroke: Improved Detection of Dense Intracranial Arteries by Varying Window Parameters and Performing a Thin-Slice Helical Scan," Neuroradiology, Vol. 44, No. 11, 2002, pp. 900906.http://dx.doi.org/10.1007/s00234-002-0848-1 